\title{
Combination of Hyaluronic Acid with Advance-Platelet Rich Fibrin to Reduce Chronic Inflammation: a study in IL-6 and Granulation Index
}

\author{
Ronald W. Kartika ${ }^{1}$, Idrus Alwi ${ }^{2}$, Mirta Hediyati Reksodiputro ${ }^{3}$, Em Yunir ${ }^{2}$, Sarwono \\ Waspadji $^{2}$, Suzzana Immanuel ${ }^{4}$, Todung Silalahi ${ }^{5}$, Saleha Sungkar ${ }^{6}$, Jusuf Rachmat ${ }^{7}$, \\ Saptawati Bardosono ${ }^{8}$, Franciscus D. Suyatna ${ }^{9, *}$

\begin{abstract}
${ }^{1}$ Department of Surgery, Faculty of Medical and Health Science, Krida Wacana Christian University, Jakarta, Indonesia; Doctoral Program in Medical Science Faculty of Medicine Universitas Indonesia; ${ }^{2}$ Department of Internal Medicine, Faculty of Medicine Universitas Indonesia - Cipto Mangunkusumo Hospital, Jakarta, Indonesia ; ${ }^{3}$ Facial Plastic Reconstructive Division, Department of Otorhinolaryngology, Faculty of Medicine, Universitas Indonesia, Cipto Mangunkusumo Hospital, Jakarta, Indonesia; ${ }^{4}$ Department of Clinical Pathology, Faculty of Medicine Universitas Indonesia - Cipto Mangunkusumo Hospital, Jakarta, Indonesia; ${ }^{5}$ Department of Internal Medicine, Krida Wacana Christian University, Jakarta, Indonesia ; ${ }^{6}$ Department of Clinical Parasitology, Faculty of Medicine Universitas Indonesia, Jakarta, Indonesia; ${ }^{7}$ Department of Thoracic Cardiac and Vascular Surgery, Faculty of Medicine Universitas Indonesia, Jakarta, Indonesia ; ${ }^{8}$ Department of Nutrition, Faculty of Medicine Universitas Indonesia, Jakarta, Indonesia; ${ }^{9}$ Department of Clinical Pharmacology, Faculty of Medicine Universitas Indonesia, Jakarta, Indonesia (Corresponding author )
\end{abstract}

Received: March 3, 2021; Revised: May 3, 2021; Accepted: September 21, 2021

\begin{abstract}
BACKGROUND: Type-2 Diabetes mellitus patients have risk of diabetic foot ulcers (DFUs) ranging from $15 \%$ to $25 \%$ with morbidity of $5 \%$ in the first 12 months, and the mortality rate at 5 years is around $42 \%$.

AIM: This research was conducted to study the novelty of a combining Hyaluronic Acid (HA) with Advanced Platelet Rich Fibrin (A-PRF) to reduce inflammation in DFUs healing through raising granulation index.

METHODS : The study used a randomized control trial design, done from July 2019-March 2020 at Gatot Soebroto Army Hospital and Koja District Hospital, Jakarta. Subjects were DFU wound area $<40 \mathrm{~cm}^{2}$. At the start of the study all subjects received the same treatment, debridement and standard wound care. Twenty subjects were recruited according to the rule of thumb and were randomly divided into two groups namely topical A-PRF + HA ( $\mathrm{n}=10)$ and $\mathrm{NaCl} 0.9 \%$ (control) groups (n $=10$ ). A part of the sample was processed into lysate and stored in $-80^{\circ} \mathrm{C}$. The rest is applied as a topical therapy in DFU base on intervention (A-PRF+HA or $\mathrm{NaCl}$ as a control). We analyzed the inflammation use swab sampling in DFU and analyze use ELISA on day- 3 and day-7. We also measured the wound area by a digital photograph and analyzed using Image $J$ at the same time.

RESULT: From this research, it was obtained 20 DFU subjects with Wagner 2 classification. We also performed analysis of IL-6 from swab topical DFU. A-PRF+HA group was significantly lower than $\mathrm{NaCl}$ (control) from swab DFU on day -3 ( p $=0,049)$ and day-7 $(p=0.041)$. In A-PRF+HA group affected on increase epithelialization process/ decrease wound area on day-3 ( $\mathrm{p}=0.016)$, day-7 ( $\mathrm{p}=0.048)$, and day-14 ( $\mathrm{p}=0.03$

CONSLUSION: With the homogeneous gel formation of A-PRF + HA, it will accelerate the formation of granulation tissue compared to conventional $\mathrm{NaCl}$ therapy through reduce inflammation by decrease IL-6 levels. In addition, the combination A-PRF + HA has the effect of accelerating the formation of epithelialization shown by decreasing wound area in intervention group.
\end{abstract}

Keywords: Diabetic Foot Ulcer- Advanced Platetelet Rich Fibrin- Hyaluronic Acid-Granulation

\subsection{Background}

One of the common complications of Diabetes mellitus has the most common complication in the form of Diabetic Foot Ulcer (DFU) with significant morbidity and mortality and requires intensive care. It is estimated that $19 \%-34 \%$ of diabetic patients have diabetic foot ulcers. According to a report by the International Diabetes Federation, 9.1-26.1 million people with diabetes develop DFU each year (Cho $\mathrm{NH}$,2018). A cohort study in the United States showed that
DFU patients increased risk of death by 2.5 times risk of death Heydar 2009).

DFU treatment options vary widely, so no single dressing is ideal for all types of wounds. The goal of the dressing should be to create a moist environment that supports granulation, autolytic processes, angiogenesis, and faster engineering of epidermal cells at the wound bed. A wide variety of dressings are available from standard treatment to adjuvant therapy. In addition, it requires

\footnotetext{
* Corresponding author e-mail: fransdsuyatna@yahoo.com.
} 
weight bearing loading, vascular assessment, control of infection and blood glucose (Everett E, 2018)

The standard care is described by Caroline C, 2016 as DFU wound care, namely local wound care with sharp debridement, daily care that increase the humidity of the wound environment. ${ }^{4}$ In standard practice in DFU care, a wide variety of agents are available and developed as adjuvant therapies. Adjuvant therapies that are developed include negative pressure wound therapy, supplement oxygen therapy, acellular bioproducts, growth factors, biologically tissue engineered (Grazul-Bilska AT, 2003).

Several growth factors have been developed for the management of DFU, including epidermal growth factors, fibroblast growth factors, endothelial vascular growth factors, granulocyte colony stimulating factors, and platelet-derived growth factors ( Doulton AJM, 2018).

There is limited data on the benefits of growth factor on wound healing in DFU, but studies evaluating plateletderived growth factor may show some benefits. Several products derived from platelets, including autologous platelet-rich plasma (PRP), used for wound healing, have been widely used by using centrifuged blood samples from patients Platelets that are processed into high concentrated suspensions will contain growth factors. Growth factors cannot be released from blood clotting by several techniques, including clotting, clotting, or clotting (Schär MO, 2015). The 2016 Cochrane Review reviewed 11 RCTs using PRP in patients with chronic wounds, although there are some drawbacks to chronic inflammatory wounds. Another developing platelet product is the leukocyte- and platelet-rich fibrin combination, which can augment growth factor and matrix protein release ( Carolline 2014). In control inflammation in DFU, a combination of A-PRF and Hyaluronic Acid is used to control inflammation (Dhurat R, 2014).

Hyaluronic acid (HA) is the main component of the extracellular matrix which plays a role in tissue regeneration (Ilio K, 2016). Specific HA receptor modulation can influence inflammation, cell migration, and angiogenesis, which are the main phases of wound healing. Other studies have revealed that combining HA with platelet-rich plasma exhibits anti-inflammatory properties in cases of knee osteoarthritis. In this review, the authors discuss the role of combined A-PRF + HA in reducing inflammation and regeneration of DFU tissue through the role of the major cellular receptors involved in HA signaling. The role of HA addition to A-PRF for healing DFU is proven by inflammation reduction, angiogenesis process, and the antioxidant properties of HA (Park D 2012)

Hyaluronic acid has anti-inflammatory properties. Likewise, A-PRF also has properties in reducing inflammation. It is hoped that A-PRF + HA can work together to reduce inflammation which is marked by a decrease in IL-6 (Ulcers DF, 2012). During chronic inflammatory process in DFU, preparation of HA can also protect and stabilize the alpha platelet granules membrane from protease enzymes so as might prevent damage to growth factors inside them.(Kartika 2021).

Until now there have been no studies comparing the combination of A-PRF + HA with A-PRF alone in reducing inflammation which affects the healing of DFU. Assessment granulation improvement can be done by using image (A. Suarez, 2020)

\section{Methods}

\subsection{Research Design and Sample}

This study has been approved by The Ethics Committee of the Faculty of Medicine Universitas Indonesia (ID 0855/UN2.F1/ETIK/2018). This open-label randomized controlled trial was conducted at Koja District Hospital and Gatot Soebroto Hospital from July 2019 to April 2020. DFU patients with wound duration of three months, Wagner-2, and ulcer size $<40 \mathrm{~cm}^{2}$ were recruited and randomly assigned into A-PRF $+\mathrm{AH}$ and control group. On day- 0 , day- 3 and day -7 , samples and photographs were taken. Samples were analyzed with ELISA and photographs were analyzed with ImageJ to calculate wound area (WA). Statistical analysis was performed using SPSS version 20.

\subsection{Material and Sample Measurement Techniques}

Measurement of IL-6 from wound surface use cotton swabs on days 0, 3 and 7 using the ELISA technique (kit) and obtained numerical data in units of pg / mL. The examination was carried out at the FKUI Integrated Laboratory. The ELISA reagent used was the Human IL-6 / ELISA Kit with the Insert Kit from LifesSpan BioSciences, Inch.Cat:LS-F4604. ELISA was done according to the manufacturer's instruction. In addition, the wounds reduction was recorded with a digital camera (Cannon Camera 48 mega pixel, China) with an accuracy of $0.1 \%$ on the $0,3,7$ and 14 days. The results of the wound photographs were processed using Image-J (Java software program) by evaluating the granulation index

\subsection{Topical A-PRF + HA preparation}

To make A-PRF, 20-40 mL of peripheral blood was taken without anticoagulant tube (RegetKit tube), then centrifuged (RegenLab centrifuge, Le Mont, Switzerland) at about $200 \mathrm{G}$ for 8 minutes. Fibrin and buffy coat are separated from erythrocytes. For the A-PRF + HA (intervention group); the process was continued by making A-PRF and HA homogenate with a ratio of 1 cc: 0.6 cc with vortex for 20 seconds. The preparation of A-PRF + HA was repeated on day-3 and day-7 and applied as a DFU topical therapy.

\subsection{Application of A-PRF + HA in DFU}

Prior to intervention, the wound was cleaned and debridement. Topical therapy was carried out by giving 1 $\mathrm{mL}$ of material for an area of $10 \mathrm{~cm}^{2}$ according to the group (group $1 \mathrm{~A}-\mathrm{PRF}+\mathrm{AH}$, group $2 \mathrm{NaCl}$ soaked gauze). The wound is covered with sterile gauze as a secondary dressing to maintain moisture.

\subsection{Evaluation of Inflammation Signs in DFU Intervention}

To assess changes in inflammation after intervention, an evaluation was carried out by looking at changes in IL6 levels (swab) on baseline, day-3 and day-7. Meanwhile, for clinical assessment, measurements of reduction of wound area from day- 0 , day-3, day-7 and day- 14 were accompanied by pain response with numeric pain score at the same time. 


\section{Result}

We recruited 20 subjects, consisting of 12 men and 8 women. The median age was 64 years in women and 61 Table 1. Subjects baseline characteristics

\begin{tabular}{|c|c|c|c|}
\hline Subject Characteristic & $\mathrm{A}-\mathrm{PRF}+\mathrm{AH}(\mathrm{n}=10)$ & Control $(n=10)$ & p-value \\
\hline Age (year) & 59,8 (SB 12,7) & 66 (SB 12.3) & 0,626 \\
\hline \multicolumn{4}{|l|}{ Sex } \\
\hline man & 5 & 3 & \\
\hline woman & 5 & 7 & \\
\hline Body Mass Index & 28,9 (SD 2,7) & 28,4 (SD 2.5) & 0,337 \\
\hline Hemoglobin (g/dL) & 12,7 (SD 1.2) & 12,05 (SD 1.4) & 0.22 \\
\hline Hematocrit (\%) & 34.1 (SD 4.3) & $33,8(\mathrm{SD} 4.5)$ & 0,145 \\
\hline Leucocyte $\left(10^{3} / \mu \mathrm{l}\right)$ & 13,30 (SD 1,08) & 9,23 (SD 1,66) & 0,985 \\
\hline Platelet $\left(10^{3} / \mu \mathrm{l}\right)$ & 354,9 (SD 167,5) & 319,9 (SD 128,4) & 0,8 \\
\hline Random Blood Glucose, mg/dL & 286,0 (SD 67.5) & 254,7 (SD 58,6) & 0,10 \\
\hline HbA1C (\%) & $11,34(\mathrm{SD} 1,30)$ & 8,5 (SD 0,72) & 0,950 \\
\hline Total Cholesterol (mg/dL) & 214,5 (SD16,9) & 202,3 (SD 38,6) & 0,096 \\
\hline Albumin (mg/dL) & 3,3 (SD 0,4) & $3,2(\mathrm{SD} 0,4)$ & 0,662 \\
\hline Anti Platelet Medication & 4 & 3 & \\
\hline Statin Medication & 3 & 4 & \\
\hline
\end{tabular}

${ }^{\mathrm{a}}$ Mean,SD, Independent $\mathrm{t}$ test

\subsection{Evaluation of IL-6 Swab DFU Receiving Intervention}

Level of IL-6 was analyzed to see the pathway of healing DFU through inflammation. The baseline IL-6 levels in the two groups were not significantly different. After two interventions, changes in levels of IL-6 ( $\Delta$ IL-6) in the A-PRF + AH group, there was a significant decrease on day( $-10.9 \mathrm{pg} / \mathrm{mg}$ protein) and day-7 of ( $-18.3 \mathrm{pg} / \mathrm{mg}$ protein), while $\Delta$ IL-6, group $\mathrm{NaCl}$ increased on day 3 of $4,3 \mathrm{pg} / \mathrm{mg}$ protein and day-7 by 35,6 pg/mg protein. The $\mathrm{A}-\mathrm{PRF}+\mathrm{AH}$ group showed a significant reduction in $\Delta$ IL-6 compared to the $\mathrm{NaCl}$ ( Control) group on day 0-7 (p $=0.015)$, according to Table 2 .

Table 2. IL-6 Levels Swab DFU Based on Intervention

\begin{tabular}{llll}
\hline Intervention & $\begin{array}{l}\text { A-PRF+HA } \\
(\mathrm{n}=10)\end{array}$ & $\begin{array}{l}\text { Control } \\
(\mathrm{n}=10)\end{array}$ & p-value \\
\hline $\begin{array}{l}\text { Before } \\
\text { treatment }\end{array}$ & $106.4(83.1-407.6)$ & $125,3(20,3-287.0)$ & 0.337 \\
Day-3 & $99.5(76.3-302.2)$ & $131,1(5,3-337,5)$ & 0.049 \\
Day-7 & $88.7(44.3-217.9)$ & $167,9(27,7-156,2)$ & $0.041^{*}$ \\
\hline$\Delta$ day- 0-3 & $-10.9(-105.4-17.7)$ & $4,3(-47.8-50,5)$ & 0.046 \\
$\Delta$ day- 0-7 & $-18.3(-189.7-44.6)$ & $35,6(-160,6-108,4)$ & 0.015
\end{tabular}

* Median (min-max), Mann Whitney test

Figrue 1 show in DFU which use topical A-PRF+HA has decrease of IL-6 swab significantly on day-3 (p $=0.049 *)$ and day-7 $\left(\mathrm{p}=0.041^{* *}\right)$, signiificantly years in men. The subjects were randomly divided into three groups (A-PRF + HA and $\mathrm{NaCl}$ ) comprised of 10 subjects per group ( Table 1)

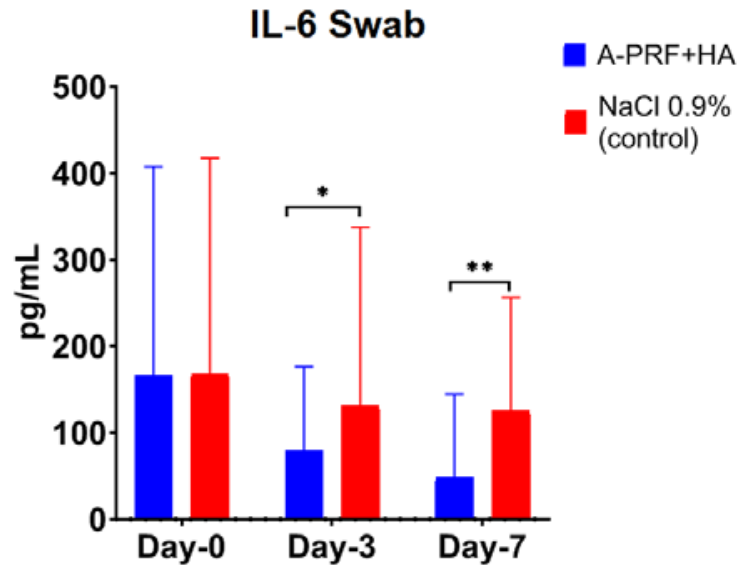

Figure 1. A-PRF +HA group decrease of IL-6 level compare with control on day-3 $\left(\mathrm{p}=0.049^{*}\right)$ and day-7 $\left(\mathrm{p}=0.041^{* *}\right)$

\subsection{Evaluation of Wound Area (WA) of DFU in A-PRF $+\mathrm{HA}$ Fibrin versus $\mathrm{NaCl}$}

To see the correlation between angiogenesis and clinical conditions, an analysis of wound area and granulation tissue growth was performed as measured by ImageJ software. The measurement of increase of epithelialization tissue changes, can be done by evaluating the woundrea (WA). The average wound area was shown in Table 3 
Table 3.Evaluation of Wound Area base on Intervention

\begin{tabular}{lllc}
\hline Intervention & $\begin{array}{l}\mathrm{A}-\mathrm{PRF}+\mathrm{HA} \\
(\mathrm{n}=10)\end{array}$ & $\begin{array}{l}\text { Control } \\
(\mathrm{n}=10)\end{array}$ & $\begin{array}{c}\mathrm{p} \\
\text { value }\end{array}$ \\
\hline Baseline & $7,0(1,9-31,9)$ & $5,2(2,0-20,6)$ & 0,848 \\
Day-3 $^{*}$ & $6,3(1,4-26,1)$ & $3,2(1,9-18,4)$ & 0,016 \\
Day-7 $^{*}$ & $5,5(1,1-25,4)$ & $2,6(1,1-17,1)$ & 0,048 \\
Day -14 & $5,0(0,9-18,6)$ & $2,3(0,3-16,2)$ & 0,030 \\
\hline$\Delta$ Day- 0-3 & $-0,5(-0,1--5,8)$ & $-1,1(-0,1--2,3)$ & 0,036 \\
$\Delta$ Day -0-7 & $-1,1(-0,3--5,9)$ & $-1,5(-0,2--3,9)$ & \\
$\Delta$ Day-0-14 & $-1,5(-0,7--13,3)$ & $-2,5(-0,5--4,4)$ & 0,045 \\
& & & 0,003 \\
\hline
\end{tabular}

* median (min-max), Mann Whitney test

Figure 2. shows the measurement, clinical evaluation and clinical photographs taken at baseline, day-3, day-7 and day-14 on different treatment groups. Here, we observed different rates of wound closure and healing, especially at day-14 compared to control group.

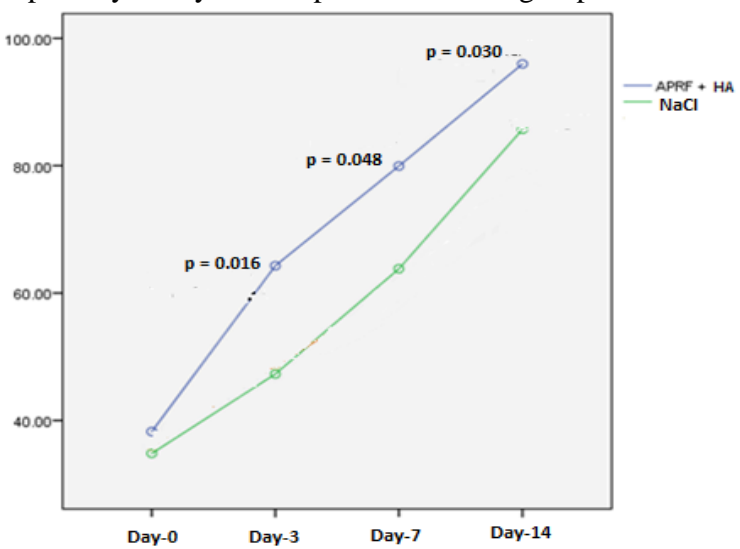

Figure 2 Wound Area of DFU at day-0, day-3, day-7 and day-14

\subsection{Evaluation of Inflammation Clinical Symptoms DFU Subjects}

We analyzed clinical symptoms such as Numeric Pain Score (NPS) and Inflammation sign in DFU. At baseline examination of the two groups in NPS evaluation was 7-8 (severe pain). After the intervention, pain scores decreased in both groups. However, there was a significant decrease of NPS on day-3 and day-7 in the A-PRF + HA group compared to $\mathrm{NaCl}$ ( contro) groups (Table 4).

Table 4. Different Numeric Pain Score (NPS) in DFU Base on Intervention

\begin{tabular}{llll}
\hline Intervention & $\begin{array}{l}\text { A-PRF + HA } \\
(\mathrm{n}=10)\end{array}$ & $\begin{array}{l}\text { Control } \\
(\mathrm{n}=10)\end{array}$ & p-value \\
\hline Baseline & $8(8-9)$ & $8(7-8)$ & 0.164 \\
Day-3 & $4(3-5)$ & $5(5-6)$ & $0.021^{*}$ \\
Day-7 & $2.5(1-3)$ & $3(3-5)$ & $0.019^{*}$ \\
Day-14 & $2.1(2-4)$ & $2(2-3)$ & $0.047^{*}$ \\
\hline
\end{tabular}

Median ( $\min -\max )$, Mann Whitney test 0 : none, $1-3=$ Mild , 4-6 : moderate : 7-9 = severe

Figure 3. show an evaluation of NPS in A-PRF + AH group compare with A-PRF group on day $-3(\mathrm{p}-0.021)^{\mathrm{a}}$, day-7 $(\mathrm{p}=0.019)^{\mathrm{b}}$, and day-7 $(\mathrm{p}=0.047)$

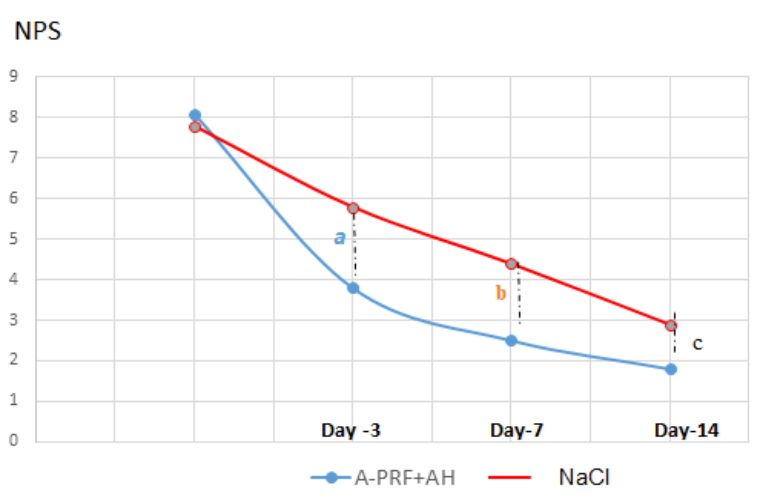

Figure 3. Numeric Pain Scale (NPS) in LKD after Intervention

We aimed to quantify the inflammatory state using an Inflammatory Score inspired by an additive effect of inflammatory sign on diabetic foot ulcers, such as redness, heat, swelling, pain, and functio laesa before and after treatment. Compared to baseline condition, the inflammation score in DFU on day-7 in A-PRF+HA showed a significant decrease in redness, heat, swelling, and pain compared to A-PRF alone. Data for these inflammation sign of DFU are shown in Table 5.

Table 5 Inflammation Sign of DFU Base on Intervention

\begin{tabular}{cccc}
\hline $\begin{array}{l}\text { Sign Of } \\
\text { Inflammation }\end{array}$ & $\begin{array}{c}\text { A-PRF+HA } \\
(\mathrm{n}=10)\end{array}$ & $\begin{array}{c}\text { Control } \\
(\mathrm{n}=10)\end{array}$ & p-value \\
\hline Redness & & \\
Day-0 & $2.9 \pm 0.5$ & $2.5 \pm 1.1$ & 0.890 \\
Day-7 & $0.1 \pm 0.03$ & $1.0 \pm 0.2$ & 0.028 \\
\hline Heat & & & \\
Day-0 & $2.8 \pm 0.1$ & $2.4 \pm 0.5$ & 0.707 \\
Day-7 & $0.3 \pm 0.1$ & $0.9 \pm 0.1$ & 0.032 \\
\hline Swelling & & & \\
Day-0 & $2.9 \pm 0.2$ & $2.5 \pm 0.3$ & 0.179 \\
Day-7 & $0.1 \pm 0.05$ & $0.7 \pm 0.2$ & 0.021 \\
\hline Pain & & & \\
Day-0 & $2.9 \pm 0.5$ & $2.7 \pm 0.6$ & 0.328 \\
Day-7 & $0.2 \pm 0.4$ & $0.8 \pm 0.1$ & 0.032 \\
\hline Functio Laesa & & & $0.9 \pm 0.1$ \\
Day-0 & $2.5 \pm 0.3$ & $2.6 \pm 0.2$ & 0.043 \\
Day-7 & $0.3 \pm 0.1$ & $0.5 \pm 0.1$ & \\
\hline Mean & & & \\
\hline
\end{tabular}

Mean (SD), independent $t$ test $0=$ none, $1=$ mild, $2=$ moderate, 3 =severe

\section{Discussions}

Treatment of diabetic foot ulcer therapy with supplementary growth factor therapy has begun to be developed in the late decades. Growth factors are needed in new cell growth and wound healing. ${ }^{14}$ change style Treatment of diabetic foot ulcers with growth factors can accelerate ulcer healing characterized by granulation tissue formation. Several growth factors influenced by the healing of DFU i.e. TGF- $\beta 1$ and $\beta 2$, IGF, and VEGF, have been used in orthopaedics, maxillofacial, periodontal fields, plastic surgery, and sports medicine because of their anti-inflammatory and antimicrobial properties (Geerlings SE et al , 2019). 
In this study, 20 DFU subjects were found with almost homogeneous basic characteristics. The use of A-PRF + HA group compared with $\mathrm{NaCl}$ (control), there was significant reduction in delta inflammation in local swabs ( $\Delta$ IL-6) in DFU on day 0-3 ( $\mathrm{p}=0.046)$ and on day 0-7 (0 $=0.015$ ).

In diabetics, there is a prolongation of the inflammatory phase in the wound healing process (Amanulla, 2020).

Several biomarkers play a role in the inflammatory process such as TGF- $\beta$ which is a pluropotent cytokine of the three isoforms in mammals such as TGF- $\beta$ 1, TGF- $\beta$ 2 , and TGF- $\beta-3$, with TGF- $\beta 1$ dominant in tissue healing, including skin. Otherwise, interleuking family also influence in inflammation in woud healing such as IL-6 and IL-1 $\beta$ (Hasan 2019).

Interleukin (IL) -6 is produced by fibroblasts, keratinocytes and macrophages of cells at the site of inflammation. IL-6 biomarkers play a role in acute to chronic inflammation by changing the nature of leukocyte infiltrates (from polymorphonuclear neutrophils to monocytes / macrophages). Additionally, IL-6 stimulates T and B cells, which support a chronic inflammatory response. Strategies that target IL-6 signaling are useful for the prevention and treatment of an effective model of diabetic foot ulcers and other chronic inflammatory diseases. IL-6 has a dual effect; to some extent, it acts as a defense mechanism but in chronic inflammation it acts as a pro-inflammatory (Gabay Cem, 2016).

In assessesment the inflammatory status of DFU, it can be both locally and systemically. In this study, a swab of DFU was carried out using a cotton swab to check for a decrease in inflammatory mediators such as IL-6. This examination is a new method that has been done for the first time. Research by Liu et al, 2002a tried to perform an MMP examination of the wound fluid Ref. Other methods used to examine biomarkers in DFU are using invasive techniques such as tissue biopsy or patch skin biopsy Ref. Research by Michael et al, 2017 has used swab examination in cases of DNA abnormalities examination in cerebral palsy cases in infants and cases of Alzheimer's Ref. The cells were obtained by means of swab or smear using a cotton bud or small brush for further analysis using the PCR technique. This method has a fairly good accuracy with a sensitivity of $93 \%$. To increase the sensitivity of the examination of other researchers, replacement of the stick swab with a soft brush is recommended (isohelix DNA / RNA buccal swab). This method can replace DNA examination with PCR of blood or hair materials. Another study at UGM used fine needle biopsy with paraffin block for detection of TGF- $\beta$ for the evaluation of biomarkers in DFU (Yang P, 2016)

\section{The use of Advance PRF in DFU healing}

In wound healing, PRF plays a crucial role in the proliferation phase by continuously releasing growth factors at the wound site and inducing viability, proliferation and differentiation of cells. PRF stimulation releases growth factors TGF- $\beta 1$ and PDGF-AA on day 5 of wound healing. A part from playing a role in accelerating wound healing through the induction of growth factors, PRF also plays an active role in inducing the proliferation and migration of skin cells through increased expression of MMP-1 and MMP-9. Platelet-rich fibrin elicited the anti-inflammatory response of macrophages in in-vitro studies. In addition, PRF has antiinflammatory activity and shifts the polarization of macrophages from M1 to the M2 phenotype (Mussano F, 2016).

Advanced platelet-rich fibrin (A-PRF) was first described in 2014 as a new concept using cell-based tissue engineering by lowering rpm while increasing the standard PRF time Ref. To obtain PRF, venous blood is drawn without adding anticoagulants. The protocol for the standard PRF (S-PRF) utilized a $2700 \mathrm{rpm}$ or $360 \mathrm{x}$ g centrifuge, for 12 minutes. In contrast to standard PRF, the APRF manufacturing technique uses a low-speed centrifuge (1500 rpm or $200 \mathrm{x}$ g for 8 minutes) because centrifugal force in speed and time which affects the distribution of growth factor cells suitable for wound healing and tissue regeneration (Nasirzade, 2020) .

Immunohistochemistry of monocytes, $\mathrm{T}$ and $\mathrm{B}$ lymphocytes, neutrophilic granulocytes, CD34, were detected around the buffy coat. Decreasing rpm while shortening the centrifugation time of A-PRF can increase the neutrophilic granulocytes in platelet fibrin concentrates more in APRF than in standard PRF, whereas in the Standard PRF (S-PRF) group, neutrophils were found on the surface of erythrocytes. Neutrophilic granulocytes play a role in the differentiation of monocytes into macrophages so that A-PRF can affect tissue regeneration, especially through monocytes / macrophages and growth factors (Gardiner E,2014).

\section{The use of Hyaluronic Acid in Wound Healing}

Isolated glycoaminoglycans has been done from the vitreous humor of a cow's eye as a hyaluronan. Hyaluronic acid is a polysaccharide with extracellular matrix components synthesized in the plasma membrane of fibroblast cells which activates many inflammatory mediators and growth factors (Greco, 1998). Hyaluronic acid recruits macrophages and modulates the inflammatory response (Ilio , 2016).

The mechanism of reducing inflammation of A-PRF + $\mathrm{AH}$ is through the pathway of changes in macrophage polarization by accelerating the polarization of M1, which has strong pro-inflammatory properties (IL-1 $\beta$, IL-6, and TNF- $\alpha$ ) to M2 (IL-10, Arginase, TGF- $\beta 1$, VEGF), which is anti-inflammatory. M1 activation was associated with inflammation, tumor resistance, and graft rejection. Conversely, M2 cell activation results in immune system regulation, cellular matrix deposition and tissue remodeling. Hyaluronic acid through the CD44 receptor in synergy with A-PRF strengthens the shift of macrophage polarization from $\mathrm{M} 1$ as a pro-inflammatory) to M2 phenotype as an anti-inflammatory. (Fujisaka, 2009)

Another advantage of HA is that it has antioxidant properties and reduces inflammation so it is widely used to treat osteoarthritis (OA). The structure of Hyaluronic acid which is contain disaccharide polymer, consisting of $\mathrm{N}$ acetyl-D-glucosamine and D-glucuronic acid, linked via $\beta$ glycosidic bonds. Hyaluronic acid inhibits the collagenase enzyme, which is the proteolysis enzyme of collagen $(\mathrm{Wu}$, 2016). Hyaluronic acid affects cell migration, cell adhesion and angiogenesis. Fibroblasts play a major role in wound healing by forming extracellular matrix components such as collagen, elastin and proteoglycans. 
Fibroblasts also play an essential role in the migration of keratinocytes from the wound edges to achieve wound closure and matrix reconstruction resulting in the maximal wound healing force of contraction (Afat et al, 2017).

\section{Combination of HA with A-PRF to Accelerate Epithelialisation}

In this study show in intervention group (Topical A$\mathrm{PRF}+\mathrm{HA}$ ) had decrease of wound area and increase granulation tissue significantly on day-3 and day-7.

Regarding the effect of HA on tissue regeneration, it is related to the interaction of HA with CD44 which drive the maintenance and establishment of collagen synthesis and normal skin function. Hyaluronic acid is present in the extracellular matrix of basal keratinocytes and the structural integrity of dermal collagen fragments and fibroblast penetration. Decreased levels of HA correlate to impaired local inflammatory response and reduced tissue repair due to degeneration of the granular tissue matrix (Fischer 2008). The combination of HA and PRP reduces pro-inflammatory cytokines and increases articular chondrocyte proliferation and chondrogenic differentiation via the HA-dependent Erk1 / 2 pathway and the PRPdependent Smad 2 / 3 pathway (Ilio , 2016). The clinical application of the combination of PRP and AH is more effective than PRP or HA alone; both are therapeutic options for osteoarthritis and chronic tendinopathy. The combined effect of PRP and AH is not fully understood. It is thought that PRP stimulates the healing process of new tissue by producing growth factors and cytokines released by platelets. The addition of HA to PRP can increase the release of growth factor on day 5 (Gardiner, 2014).

Combination of AH with PRF stimulated growth factors such as TGF- $\beta$, significantly increasing the proliferation index and collagen deposition ( Ilio, 2016).
AH also interacts with the TGF-ß1 transformation of PRF thereby protecting growth factors from degradation of tryptic and collagen by protease enzymes (Afat, 2017).Combination of AH with L-PRF reduced edema after oral surgery of the 3rd molar although through HA linking wih ICAM, VCAM receptor. This link will reduce vascular leakage of neutrophil and reduce edema. (Fathi, 2012)

Hyaluronic acid affects three main receptors in tissue regeneration's modulation, namely migration, proliferation and activation of keratinocyte cells (CD44). This is done to restore the epidermis, fibroblast migration (RHAMM), control of inflammation and neoangiogenesis (ICAM-1), as well as promotion of ECM deposits such as collagen fibers that contribute to wound healing (Park, 2012). Wound healing begins with a complex integration process through cellular, physiological, and biochemical processes, such as inflammation, cell migration, and proliferation. Interleukin-6 is a multifunctional cytokine that regulates the inflammatory response of the wound healing process in a timely manner. Hyaluronic acid is an important component of ECM contributing significantly to cell proliferation and migration. The combination of PRF and AH provides a synergistic effect on wound healing cell migration with ERK (extracellular signal) activation (Wu, 2016).

In this study, A-PRF corresponded to high concentrations of platelets in liquid fibrinogen which contains growth factors required for soft tissue regeneration. The low speed and low time centrifugation protocol (200 G, 8 minutes) lead to enhance the healing of chronic diabetic foot wounds. The addition of HA to A-PRF will further induce the release of growth factors (Kartika RW, 2020).

Here is the proposed mechanism of A-PRF + HA in reducing inflammation and increase granulation in wound healing (Figure 4).

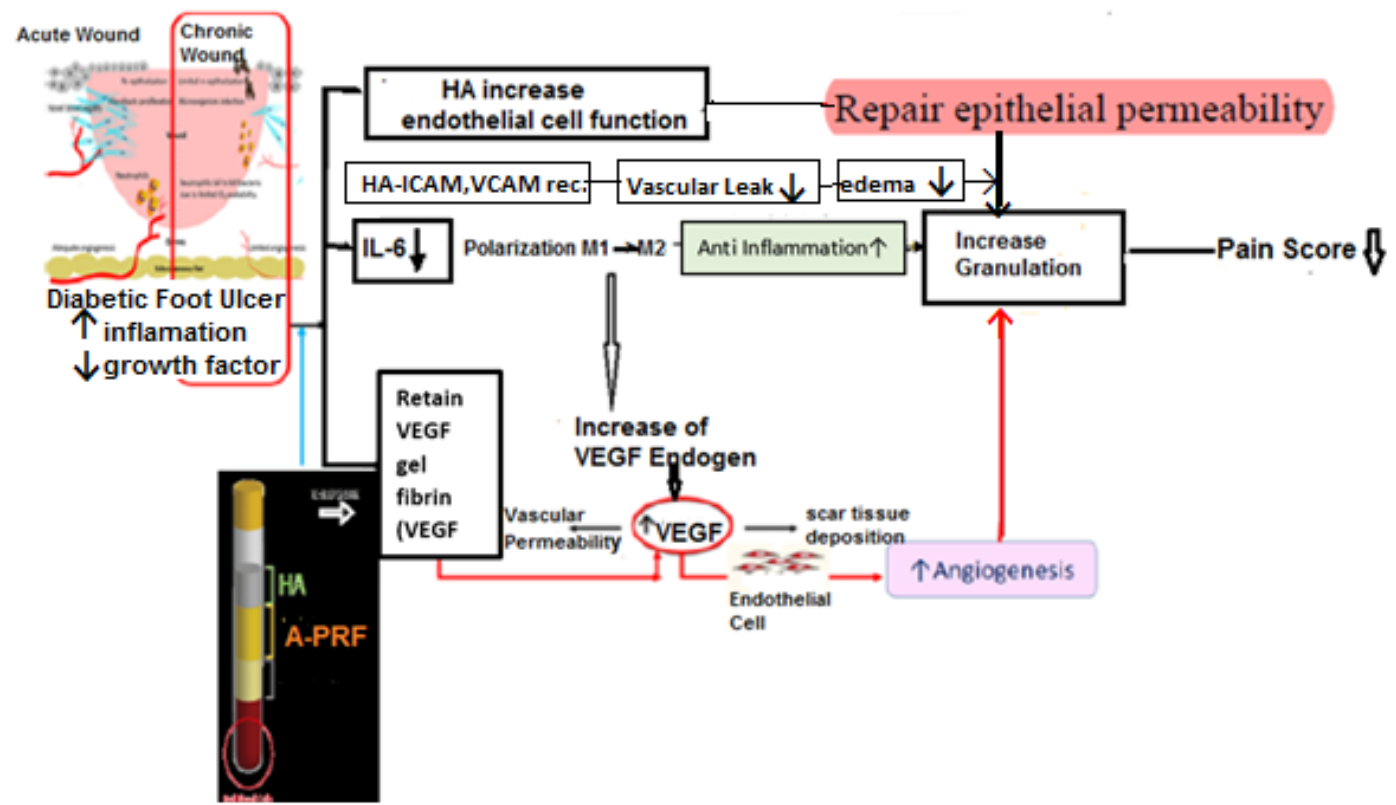

Figure 4. Proposed mechanism of A-PRF HA increase Granulation in DFU healing

\section{Conclusion}

The combination of A-PRF and HA increases the granulation and epithelialization in DFU healing by reducing the inflammation state which will induce the angiogenesis process. Clinically, the application of A-PRF and HA combination showed to reduce pain better than the control $\mathrm{NaCl}$ in $\mathrm{DFU}$ patients. 


\section{Limitation Study}

The subject in this study only 20 patient and the evaluation of inflammation was only the use of Interleukin -6 and clinical inflammation preview. We need more subjects for evaluation with more variants of inflammation biomarker.

\section{Acknowledge}

This study was a dissertation of Program Doctoral Medical Science Universitas Indonesia.

\section{Conflict of Interest} Jakarta.

This study was funded by Universitas Indonesia,

\section{References}

Cho NH. 2018. IDF Diabetes Atlas : Global estimates of diabetes prevalence for 2017 and projections for 2045. Diabetes Res Clin Pract, 138:271-81. doi:10.1016/j.diabres.2018.02.023

Heydari I. 2010. International Journal of Diabetes Mellitus Chronic complications of diabetes mellitus in newly diagnosed patients. Int $\mathrm{J}$ Diabetes Mellit, 2(1):61-3. doi:10.1016/j.ijdm.2009.08.001

Everett E and Mathioudakis N. 2018. Update on management of diabetic foot ulcers. Ann N Y Acad Sci, 11(1):153-65. doi:10.1111/nyas.13569

Caroline C.L.M and Naves .2016, The Diabetic Foot: A Historical Overview and Gaps in Current Treatment, Adv.W Care, 5 (5),1-7

Grazul-Bilska AT. 2003. Wound healing: The role of growthfactors.DrugsToday,39(10): 787-800. doi:10.1358/dot2003.39

Doulton AJM. 2018. Diagnosis and management of diabetic foot complications.Current Diabetes Update, (2):1-20. doi:10.2337/DB20182-1

Schär M. 2015. Platelet-rich Concentrates Differentially Release Growth Factors and Induce Cell Migration In Vitro. Clin Orthop Relat Res, 473(5):1635-43. doi:10.1007/s11999-015-4192-9

Dhurat R and Sukesh M, 2104. Principles and methods of preparation of platelet-rich plasma: A review and author's perspective. J Cutan Aesthet Surg,7(4):189-95. doi:10.4103/0974-2077.150734

Vokurka J, 2016. Concentrations of growth factors in platelet-rich plasma and platelet-rich fibrin in a rabbit model. Vet Med (Praha), 61(10):567-70. doi:10.17221/24/2016-VETMED

Iio K. 2016. Hyaluronic acid induces the release of growth factors from platelet-rich plasma. Asia-Pacific J Sport Med Arthrosc Rehabil Technol, 4(3) : 17-32 doi:10.1016/j.asmart.2016.01.001

Fathi WK. 2012. The Effect of Hyaluronic Acid and Platelet Rich Plasma on Soft Tissue Wound Healing : An Experimental Studyon Rabbits. Al-Rafidain Dent J, 12(3):115-25.

Ulcers DF. 2012.Role of Interleukin-6 ( IL-6 ) and Indicators of Inflammation in the Pathogenesis of. Aust. J. Basic \& Appl. Sci., 6(6):430-5.

Suarez. 2020, An image J plugin for the high throughput image analysis of in vitro scratch wound healing assays,Plos One, 15(7):1-14, doi. org/10.1371 /journal.pone.0232565
Geerlings SE and Hoepelman AI. 2019. Immune dysfunction in patients with diabetes mellitus (DM). FEMS Immunol Med Microbiol, 26(3):259- $65 . \quad$ doi: $10.1111 /$ j.1574695X.1999.tb01397.x

Harris M.2008. Challenges in diabetes management. Aust Fam Physician 37(9):716-20. doi:10.1186/1744-8603-9-63

Agrawal M .2015. Platelet Rich Fibrin and its Applications in Dentistry- A Review Article. Int J Clin Exp Med, 8(5): 7922-9

Gabay Cem.2006. Interleukin-6 and chronic inflammation, Arthritis Res Ther, 8(2):3-8 doi: 10.1186/ar1917

Liu RAN .2002. Ratios of activated matrix metalloproteinase-9 to tissue inhibitor of matrix metalloproteinase- 1 in wound fluids are inversely correlated with healing of pressure ulcers.. Wound Repair Regen , 1(2):26-37. doi 10.1046/j.1524475x.2002.10903.x

Michael GL .2017. Difference of DNA Quantity Extracted from Buccal Swab with Different Amount of Swab. J Kedokt Diponegoro, 6(2):443-50.

Yang P.2016. Compromised wound healing in ischemic type 2 diabetic rats. PLoS One , 11(3):1-19 doi:10.1371. /journal pone. 0152068

Gill SE and Parks WC . 2009. Metalloproteinases and Their Inhibitors: Regulators of Wound Healing, 40(206):1334-47. doi:10.1016/j.biocel.2007.10.024

Mussano F.2016. Cytokine, chemokine , and growth factor profile of platelet-rich plasma. Platelets , 27(5):467-71. doi :10.3109. /09537104.2016.1143922

Nasirzade J.2020. Platelet-Rich Fibrin Elicits an AntiInflammatory Response in Macrophages In Vitro. J Periodontol, 91(2):244-52. doi: 10.1002/JPER.19-0216.

Gardiner E. 2014.A Systems Biology Approach to Blood. Exp Hematol,12(844):201-25 doi:10.1007/978-1-4939-2095-2

Afat IM.2017. Effects of leukocyte- and platelet-rich fibrin alone and combined with hyaluronic acid on pain, edema, and trismus after surgical extraction of impacted mandibular third molars. J Oral Maxillofac Surg, 76(5):926-32 doi: 10.1016/j.joms.2017.12.005.

Park D. 2012.Hyaluronic acid promotes angiogenesis by inducing RHAMM-TGF $\beta$ receptor interaction via CD44-PKC 8 . Mol Cells, 33(6):563-74. doi:10.1007/s10059-012-2294-1

Wu X.2016. Src promotes cutaneous wound healing by regulating MMP-2 through the ERK pathway.International $\mathrm{J}$ of Molecular Med, (37)3: 639-48. doi:10.3892/ijmm.2016.2472

Amanulla A.2020. Assosiation of hs-CRP with Diabetic and nonDiabetic Individuals,JJBS, 2(1):1-17

Snur Muhammaed Amir Hassan.2019. Anti-inflammatory and Anti-proliferative Activity of Coconut Oil against Adverse Effects of UVB on Skin of Albino Mice, JJBS, 13(3):295-302

Greco RM, Iocono JA, Ehrlich HP. Hyaluronic acid would stimulates human fibroblast proliferation within a collagen matrix. J Cell Physiol. 1998;177(3):465-73. doi:10.1002/(SICI)1097-4652

Fujisaka S, Usui I, Bukhari A, Ikutani M, Oya T, Kanatani Y et al (2009). Regulatory Mechanisms for Adipose Tissue M1 and M2 Macrophages in Diet-Induced Obese Mice.;58:2574-82

Fisher GJ, Varani J, Voorhees JJ, 2008) . Looking older: Fibroblast Collapse and Therapeutic Implication . Arch Dermatol.; 144(5): 666-672.

Kartika RW, Alwi I, Yunir EM,Waspadji S, Suyatna F,Bardosono S, Immanuel S, Rachmat J, Silallahi T, Reksodiputro M, Sungkar S (2011) Efficacy of Combining Hyaluronic Acid and PlateletRich Fibrin in Diabetic Foot Ulcer, JJBS ; 14(3):607-611. 